\title{
ESTIMATES FOR THE NUMBER OF REAL-VALUED CONTINUOUS FUNCTIONS
}

\author{
BY \\ W. W. COMFORT $\left({ }^{1}\right)$ AND ANTHONY W. HAGER $\left({ }^{1}\right)$
}

\begin{abstract}
It is a familiar fact that $|C(X)| \leqq 2^{\delta X}$, where $|C(X)|$ is the cardinal number of the set of real-valued continuous functions on the infinite topological space $X$, and $\delta X$ is the least cardinal of a dense subset of $X$. While for metrizable spaces equality obtains, for some familiar spaces-e.g., the one-point compactification of the discrete space of cardinal $2 \times 0$ - the inequality can be strict, and the problem of more delicate estimates arises. It is hard to conceive of a general upper bound for $|C(X)|$ which does not involve a cardinal property of $X$ as an exponent, and therefore we consider exponential combinations of certain natural cardinal numbers associated with $X$. Among the numbers are $w X$, the least cardinal of an open basis, and $w c X$, the least $\mathfrak{m}$ for which each open cover of $X$ has a subfamily with $\mathfrak{m}$ or fewer elements whose union is dense. We show that $|C(X)| \leqq(w X)^{w c X}$, and that this estimate is best possible among the numbers in question. (In particular, $(w X)^{w c X} \leqq 2^{\delta X}$ always holds.) In fact, it is only with the use of a version of the generalized continuum hypothesis that we succeed in finding an $X$ for which $|C(X)|<(w X)^{w c X}$.
\end{abstract}

Three further points warrant mention. (a) As a corollary of the result discussed above, we find that $(w X)^{w c X}=(w \beta X)^{w c X}$, where $\beta X$ denotes the Stone-Čech compactification of $X$; this equation can be taken as a means of estimating $w \beta X$ in terms of $X$. (b) For one of the examples indicating the delicacy of our result, we use a product space whose salient features are isolated via the theorem: if $\delta X_{\alpha} \leqq \mathfrak{m}$ for each $\alpha$, then in $\Pi_{\alpha} X_{\alpha}$ each family of pairwise disjoint open sets has $\mathfrak{m}$ or fewer members. This is proved by applying to the general cardinal $\mathfrak{m}$ the ideas used by Marczewski in [M] for $\mathfrak{m}=\boldsymbol{\aleph}_{0}$. (c) The main theorem, 2.2, and its lemma, 2.1, require no separation axioms whatever. We take as a standing hypothesis elsewhere throughout this paper that each of the spaces considered is completely regular and Hausdorff; furthermore, each example we construct is completely regular and Hausdorff. The case of finite spaces is disposed of in $\S 1$, and thereafter all spaces are infinite.

Presented to the Society, August 27, 1969; received by the editors August 6, 1969.

AMS Subject Classifications. Primary 5428, 5440; Secondary 0430, 4625, 5452, 5453.

Key Words and Phrases. Real-valued continuous functions, weight, density character, Stone-Cech compactification, Marczewski Theorem, Šanin Theorem, continuum hypothesis.

${ }^{1}$ ) The authors gratefully acknowledge support received from the National Science Foundation under grants NSF-GP-8357 and NSF-GP-18825. Further, the second author's research was partially supported by a grant from Wesleyan University.

Copyright 두 1970, American Mathematical Society 
1. Notation, elementary relations, and a summary. We now define and discuss briefly the cardinal numbers associated with a topological space which will be of interest to us, and summarize in tabular form much of the contents of the paper.

1.1 Definition. Let $X$ be a topological space.

$p X$, the pseudocompactness number, is the least cardinal $\mathfrak{m}$ for which each locally finite family of open sets of $X$ has $\mathfrak{m}$ or fewer members.

$w c X$, the weak covering number, is defined in the introduction.

$o X$, the cellular number, is the least cardinal $m$ for which each family of pairwise disjoint open sets in $X$ has $\mathfrak{m}$ or fewer members.

$\delta X$, the density character, is defined in the introduction.

$\pi w X$, the $\pi$-weight, is the least cardinal of a $\pi$-basis. A $\pi$-basis for $X$ is a family $\mathscr{S}$ of nonvoid open sets such that each nonvoid open set contains a member of $\mathscr{S}$. $w X$, the weight of $X$, is defined in the introduction.

Of course, the numbers $\delta X$ and $w X$ are familiar. For the others, we cite some occurrences in the literature (with no claim to completeness) so that the interested reader might ascertain some of the uses to which these numbers have been put. $p X:$ [I, Chapter $\mathrm{VII}],\left[\mathrm{H}_{2}\right],[\mathrm{N}] . w c X:[\mathrm{F}],[\mathrm{CHN}],\left[\mathrm{H}_{2}\right],[\mathrm{Hi}] . o X:[\mathrm{Ef}],[\mathrm{ET}]$. $\pi w X:[\mathrm{Pm}],[\mathrm{O}],[\mathrm{Ef}]$. It should be noted that in these papers, the definitions often differ slightly from 1.1, and the terminology and notation are often completely different.

The reader might note that we have made no mention of the "covering number" of a space, the least cardinal $\mathfrak{m}$ for which each open cover has a subcover with $\mathfrak{m}$ or fewer members. This is because, in our context, the number is essentially uninteresting; this is established in $§ 8$. Aside from this, and an ancillary use of this number in $\$ 6$, the number will not be mentioned.

For $S$ a set, $|S|$ denotes the cardinality of $S$.

Observe that for a finite space $X$ (assumed Hausdorff, recall) $p X=w c X=c X$ $=o X=\delta X=\pi w X=w X=|X|$, and $|C(X)|=2^{\aleph_{0}}$. Henceforth, all spaces are infinite, so that (clearly) all the numbers $p X$ through $w X$ are infinite.

1.2 Proposition. For any space $X$,

$$
p X \leqq w c X \leqq o X \leqq \delta X \leqq \pi w X \leqq w X \leqq 2^{\delta X} .
$$

Proof. (1) Let $\mathscr{U}$ be a locally finite family of open sets, and let $\mathscr{V}$ be an open cover each member of which meets only finitely many members of $\mathscr{U}$. Extract $\mathscr{W} \subset \mathscr{V}$ with $|\mathscr{W}| \leqq w c X$ and $\bigcup \mathscr{W}$ dense. For $W \in \mathscr{W}$, let

$$
\mathscr{U}(W)=\{U \in \mathscr{U}: U \cap W \neq \varnothing\} .
$$

Each $\mathscr{U}(W)$ is finite, and $\mathscr{U}=\bigcup\{\mathscr{U}(W): W \in \mathscr{W}\}$, so that

$$
|\mathscr{U}| \leqq \sum\{|\mathscr{U}(W)|: W \in \mathscr{W}\} \leqq(w c X) \cdot \aleph_{0}=w c X .
$$

It follows that $p X \leqq w c X$. 
(2) Let $\mathscr{U}$ be an open cover. There is, by Zorn's Lemma, a family $\mathscr{V}$ whose members are open and pairwise disjoint, each member being contained in some member of $\mathscr{U}$, and which is maximal with respect to these properties. Evidently $|\mathscr{V}| \leqq o X$, and by maximality $\cup \mathscr{V}$ is dense. Let $\mathscr{W}$ be a subfamily of $\mathscr{V}$ obtained by picking, for each $V \in \mathscr{V}$, a member of $\mathscr{U}$ containing $V$. Then $\bigcup \mathscr{W}$ is dense, $|\mathscr{W}| \leqq o X$, and $w c X \leqq o X$ follows.

(3), (4), and (5) are trivial.

(6) Let $\mathscr{R}$ be the collection of "regular open" sets, those $U$ with $U=$ int cl $U$. $\mathscr{R}$ is a basis, so $w X \leqq|\mathscr{R}|$. Let $D$ be a dense set with $|D|=\delta X$. Since different members of $\mathscr{R}$ differ by at least an open set, the map $U \rightarrow U \cap D \in 2^{\delta X}$ is one-to-one on $\mathscr{R}$; thus $w X \leqq|\mathscr{R}| \leqq 2^{\delta X}$.

The following table summarizes much of the further content of the paper.

\begin{tabular}{|c|c|c|c|c|c|c|}
\hline \multicolumn{6}{|c|}{ Cardinals $a$ and $b$ for which $|C(X)| \leqq a^{b}$} \\
\hline$b$ & $p X$ & $w c X$ & $o X$ & $\delta X$ & $\pi w X$ & $w X$ \\
\hline$p X$ & $\mathrm{~F}$ & $\mathrm{~F}$ & $\mathrm{~F}$ & $\mathrm{~T}$ & $\mathrm{~T}$ & $\mathrm{~T}$ \\
\hline$w c X$ & $\mathrm{~F}$ & $\mathrm{~F}$ & $\mathrm{~F}$ & $\mathrm{~T}$ & $\mathrm{~T}$ & $\mathrm{~T}$ \\
\hline$\delta X$ & $\mathrm{~F}$ & $\mathrm{~F}$ & $\mathrm{~F}$ & $\mathrm{~T}$ & $\mathrm{~T}$ & $\mathrm{~T}$ \\
\hline$\delta X$ & $\mathrm{~F}$ & $\mathrm{~F}$ & $\mathrm{~F}(4.1)$ & $\mathrm{T}$ & $\mathrm{T}$ & $\mathrm{T}$ \\
\hline$w w X$ & $\mathrm{~F}$ & $\mathrm{~F}(4.2)$ & $\mathrm{T}(2.4)$ & $\mathrm{T}$ & $\mathrm{T}$ & $\mathrm{T}$ \\
\hline$w X$ & $\mathrm{~F}(4.4)$ & $\mathrm{T}(2.2)$ & $\mathrm{T}$ & $\mathrm{T}$ & $\mathrm{T}$ & $\mathrm{T}$ \\
\hline
\end{tabular}

TABLE 1.3

The entry in row $a$ and column $b$ is the assertion of the truth (designated T) or falsity (designated F) of the statement

$$
\text { “ }|C(X)| \leqq a^{b} \quad \text { for every space } X . "
$$

Thus, for example, in the $p X$-row and $\delta X$-column we find " $\mathrm{T}$ ", since $|C(X)|$ $\leqq 2^{\delta X} \leqq p X^{\delta X}$ always holds. (This, by the way, is reproved in 2.4 below.) In fact, all the T's in the $\delta X-, \pi w X$ - and $w X$-columns follow from this: both the row coordinates and column coordinates are listed in the weakly increasing order given by 1.2, and hence each entry below or to the right of a " $\mathrm{T}$ " entry is itself a " $T$ " entry, and each entry above or to the left of an " $F$ " entry is itself an " $F$ " entry. Thus, to fill in the entire table, it suffices to establish just those entries which 
are followed by numbers; these numbers indicate where the proof (or example) is to be found in the sequel.

2. The upper bounds for $|C(X)|$. We begin with a lemma which will yield that $(w X)^{w c X}$ and $(\pi w)^{o X}$ (as well as $2^{\delta X}$ ) are upper bounds for $|C(X)|$, though it will develop that the first of these never exceeds the second. It seems likely that the lemma has other applications as well.

2.1 LEMMA. Let $\mathfrak{m}$ be a cardinal number and $\Phi$ a family of collections of subsets of the space $X$, such that if $\mathscr{A} \in \Phi$ then $|\mathscr{A}| \leqq \mathfrak{m}$ and $\bigcup \mathscr{A}$ is dense in $X$. Suppose that for each positive integer $n$ and each $f$ in $C(X)$ there is an element $\mathscr{A}$ of $\Phi$ for which $\operatorname{osc}_{A} f<1 / n$ whenever $A \in \mathscr{A}$. Then $|C(X)| \leqq|\Phi| \aleph_{0} \cdot 2^{\mathfrak{m}}$.

Proof. Let $f \in C(X)$. For each $n$, choose $\mathscr{A}(f, n) \in \Phi$ as prescribed by the hypotheses. For $A \in \mathscr{A}(f, n)$, let $s_{(f, n)}(A)$ be a rational number for which $\left|f(x)-s_{(f, n)}(A)\right|$ $<1 / n$ whenever $x \in A$. Observe that $s_{(f, n)}$ is a rational-valued function defined on $\mathscr{A}(f, n)$, i.e., $s_{(f, n)} \in \mathbf{Q}^{\mathscr{A}(f, n)}$ (with $\mathbf{Q}$ denoting the rational numbers). Let $S_{n}(f)$ be the ordered pair $\left(\mathscr{A}(f, n), s_{(f, n)}\right)$, an element of $\Phi \times \mathbf{Q}^{\mathscr{A}(f, n)} \subset \bigcup\left\{\Phi \times \mathbf{Q}^{\mathscr{A}}: \mathscr{A} \in \Phi\right\}$. Let $\varphi(f)$ be the sequence $\left(S_{1}(f), S_{2}(f), \ldots\right)$, so that

$$
\varphi(f) \in \prod_{n}\left[\bigcup\left\{\Phi \times \mathbf{Q}^{\mathscr{A}}: \mathscr{A} \in \Phi\right\}\right] .
$$

The cardinality of this last set does not exceed

$$
\left(|\Phi| \cdot\left|\Phi \times \mathbf{Q}^{\mathfrak{m}}\right|\right)^{\aleph_{0}}=\left(|\Phi|^{2} \cdot\left|\mathbf{Q}^{\mathfrak{m}}\right|\right)^{\aleph_{0}}=|\Phi|^{\aleph_{0}} \cdot\left(\boldsymbol{\aleph}_{0}^{\mathfrak{m}}\right)^{\aleph_{0}}=|\Phi|^{\aleph_{0}} \cdot 2^{\mathfrak{m}}
$$

It remains to show that the mapping $f \rightarrow \varphi(f)$ is one-to-one on $C(X)$. So suppose $f \neq g$, say $g$ is less than $f$ somewhere. Then there are a positive integer $n$, a real number $r$, and a nonvoid open set $U$ for which

$$
f(x) \geqq r+2 / n \text { and } g(x) \leqq r-2 / n
$$

whenever $x \in U$. If $\varphi(f)=\varphi(g)$, then in particular

$$
\left(\mathscr{A}(f, n), s_{(f, n)}\right)=S_{n}(f)=S_{n}(g)=\left(\mathscr{A}(g, n), s_{(g, n)}\right) .
$$

Since $\bigcup \mathscr{A}(f, n)$ is dense in $X$, there is $A \in \mathscr{A}(f, n)$ with $A \cap U \neq \varnothing$. So if $p \in A \cap U$, then

$$
f(p) \geqq r+2 / n \text { and }\left|f(p)-s_{(f, n)}(A)\right|<1 / n \text {, }
$$

which implies $s_{(f, n)}(A) \geqq r+1 / n$. But since $\mathscr{A}(f, n)=\mathscr{A}(g, n)$, we also have

$$
g(p) \leqq r+2 / n \text { and }\left|g(p)-s_{(g, n)}(A)\right|<1 / n,
$$

which implies $s_{(g, n)}(A) \leqq r+1 / n$. Since $s_{(g, n)}(A)=s_{(f, n)}(A)$, we have a contradiction.

Observe this. If $D$ is dense in $X$ and $|D|=\delta X$, and $\Phi$ is taken to be the single collection $\{\{x\}: x \in D\}$, then applying the lemma yields $|C(X)| \leqq(\delta X)^{\aleph_{0}} \cdot 2^{\delta X}=2^{\delta X}$, the classical estimate. Furthermore, if $X$ is discrete, so that $D=X,|C(X)|=2^{\delta X}$, 
showing that under the hypotheses of 2.1 , the upper bound given there cannot be lowered.

2.2 TheOREM. For any $X,|C(X)| \leqq(w X)^{w c X}$.

Proof. Let $\mathscr{B}$ be an open basis for $X$ with $|\mathscr{B}|=w X$. We shall apply Lemma 2.1 with

$$
\Phi=\{\mathscr{A} \subset \mathscr{B}:|\mathscr{A}| \leqq w c X \text { and } \bigcup \mathscr{A} \text { is dense }\} .
$$

Note that $|\Phi| \leqq(w X)^{w c X}$, so that 2.1 will yield:

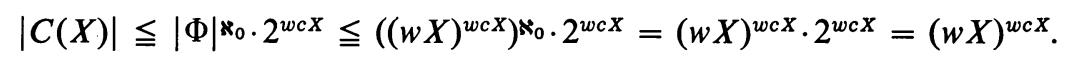

That $\Phi$ satisfies the hypotheses of 2.1 is easily checked: given $f$ and $n$, choose for each $x \in X$ a set $B_{x} \in \mathscr{B}$ with $x \in B_{x}$ and $\operatorname{osc}_{B_{x}} f<1 / n$; let $\mathscr{A}$ be a subfamily of $\left\{B_{x}: x \in X\right\}$ with $|\mathscr{A}| \leqq w c X$ and $\bigcup \mathscr{A}$ dense; clearly, $\mathscr{A} \in \Phi$.

2.3 REMARK. In $\left[\mathrm{H}_{1}\right]$, it is shown by special means that for a Lindelöf space $X$ (for which $w c X=\aleph_{0}$, of course), $|C(X)| \leqq(w X)^{\aleph_{0}}$. (In fact, it is shown that equality holds. See $\S 7$ below.) In a note added in proof in $\left[\mathrm{H}_{1}\right]$, a rudimentary and somewhat erroneous version of the proof of 2.2 is sketched.

The result for Lindelöf spaces itself generalizes the result for compact spaces, essentially due to Smirnov in [S]. See $\S 7$ below.

Lemma 2.1 also yields a proof that $|C(X)| \leqq(\pi w X)^{o X}$, as follows. Choose a $\pi$-base $\mathscr{B}$ for $X$ with $|\mathscr{B}|=\pi w X$, and let $\Phi \equiv\{\mathscr{A} \subset \mathscr{B}: \cup \mathscr{A}$ is dense and the members of $\mathscr{A}$ are pairwise disjoint $\}$. Evidently, $|\Phi| \leqq(\pi w X)^{o X}$. The hypotheses of 2.1 are verified as in 2.2 , and using 2.1 , the result follows.

However, this bound can be deduced from 2.2. We now describe how, and show that our bounds never exceed the classical one, $2^{\delta x}$.

2.4 TheOREM. For any $X,|C(X)| \leqq(w X)^{w c X} \leqq(\pi w X)^{o X} \leqq 2^{\delta X}$.

Proof. The first inequality is 2.2. The second follows from the inequality $w X \leqq(\pi w X)^{o X}$, stated in [Ef, Lemma 2], and the fact that w $X \leqq o X$ (1.2). Since no proof of the former is given in [Ef], we sketch one.

Let $\mathscr{B}$ be a $\pi$-base with $|\mathscr{B}|=\pi w X$, and let $\mathscr{R}$ be the collection of "regular open" sets, i.e., sets $U$ with $U=$ int cl $U$. Since $\mathscr{R}$ is a basis, $w X \leqq|\mathscr{R}|$. Now, for $U \in \mathscr{R}$, let $\mathscr{B}(U)$ be a subfamily of $\mathscr{B}$ with $\bigcup \mathscr{B}(U) \subset U$, whose members are pairwise disjoint and which is maximal with respect to these properties. The maximality makes $\bigcup \mathscr{B}(U)$ dense in $U$, and this makes the map $\grave{U} \rightarrow \mathscr{B}(U)$ one-to-one on $\mathscr{R}$. Then $|\mathscr{R}| \leqq(\pi w X)^{o X}$ follows.

Finally, using 1.2, we have $(\pi w X)^{o X} \leqq(w X)^{\delta X} \leqq\left(2^{\delta X}\right)^{\delta X}=2^{\delta X}$. Thus 2.4 is proved.

There is a trivial lower bound for $|C(X)|$, namely $w X$ : the sets $\{x \in X: f(x) \neq 0\}$, for $f \in C(X)$, form a basis (for completely regular $X$ ). If we assume that there are no cardinal numbers strictly between $\delta X$ and $2^{\delta X}$, then it follows that among the inequalities

$$
\delta X \leqq|C(X)| \leqq(w X)^{w c X} \leqq(\pi w X)^{o X} \leqq 2^{\delta X}
$$


exactly one must be strict. The examples later in this paper show that a strict inequality can occur anywhere in the string.

3. Remarks on the cellular number of a product. For use in one of the examples in $\S 4$, we now give a proof that $o\left(\prod\left\{X_{\alpha}: \alpha \in A\right\}\right) \leqq \mathfrak{m}$ if $\delta X_{\alpha} \leqq \mathfrak{m}$ for each $\alpha \in A$. This generalizes the well-known theorem of Marczewski which concerns the product of separable spaces. (See also 2.7 of [O].) For regular cardinals $m$, the general theorem follows readily from a theorem attributed to Šanin $\left(\left[\breve{S}_{1}\right],\left[\breve{\mathbf{S}}_{2}\right]\right)$ by Ross and Stone [RS]. Our proof uses the techniques used for $m=\boldsymbol{\aleph}_{0}$ in $[\mathrm{RS}$, Theorem 2].

We begin by recalling the Hewitt-Marczewski-Pondiczery Theorem. (See [He], $[\mathrm{M}],[\mathrm{Pz}]$.)

3.1 TheOREM. Let $X=\prod_{\alpha \in A} X_{\alpha}$, where $|A| \leqq 2^{\mathfrak{m}}$ and $\delta X_{\alpha} \leqq \mathfrak{m}$ for each $\alpha$ in $A$. Then $\delta X \leqq \mathfrak{m}$.

3.2 Theorem. Let $X=\prod_{\alpha \in A} X_{\alpha}$, where $\delta X_{\alpha} \leqq \mathfrak{m}$ for each $\alpha$ in $A$. Then $o X \leqq \mathfrak{m}$.

Proof. Suppose, instead, that there is a collection $\left\{U^{i}: i \in I\right\}$ of nonvoid open subsets of $X$ for which $|I|=\mathfrak{m}^{+}$and $U^{i} \cap U^{j}=\varnothing$ whenever $i$ and $j$ are distinct elements of $I$. We may suppose that each of the sets $U^{i}$ has the form

$$
U^{i}=\left(\prod_{\alpha \in F_{i}} U_{\alpha}^{i}\right) \times\left(\prod_{\alpha \in A \mid F_{i}} X_{\alpha}\right)
$$

where for each $i$ in $I$ the set $F_{i}$ is a finite subset of $A$ and each of the sets $U_{\alpha}^{i}$ is open in $X_{\alpha}$. Writing $F=\bigcup_{i \in I} F_{i}$ and defining

$$
V^{i}=\left(\prod_{\alpha \in F_{i}} U_{\alpha}^{i}\right) \times\left(\prod_{\alpha \in F \backslash F_{i}} X_{\alpha}\right)
$$

we notice that the sets $V^{i}$ are open in the product space $\prod_{\alpha \in F} X_{\alpha}$, and that $\delta\left(\prod_{\alpha \in F} X_{\alpha}\right) \leqq \mathfrak{m}$ using Theorem 4.1 and the fact that $\left.|F| \leqq \mathfrak{m}^{+} \leqq 2^{\mathfrak{m}}\right)$.

The desired contradiction is achieved by observing that the $\mathfrak{m}^{+}$sets $V^{i}$ are pairwise disjoint. (Indeed, if $i \neq j$ and $p \in V^{i} \cap V^{j}$, then

$$
\left.\{p\} \times \prod_{\alpha \in A \backslash F} X_{\alpha} \subset U^{i} \cap U^{j} .\right)
$$

From the theorem just proved there follows the possibility of constructing spaces $X$ for which $\delta X$ is an arbitrarily large number (established in advance) while $o X$, and a fortiori $w c X$, are small. Specifically, we have the following.

3.3 Corollary. Let $X=\prod_{\alpha \in A} X_{\alpha}$, where $\delta X_{\alpha} \leqq \mathfrak{m}$ for each $\alpha$ in $A$. If $Y$ is any dense subspace of $X$, then $w c Y \leqq o Y \leqq \mathfrak{m}$.

Proof. The first inequality is given by (2) of Lemma 1.2. The second follows from 3.2 and the fact that $o Y=o X$ whenever $Y$ is dense in $X$ (a result independent of the structural hypothesis $X=\prod_{\alpha \in A} X_{\alpha}$ ). To establish this fact let $Y$ be dense in an 
arbitrary space $X$ and suppose first that $\mathscr{U}$ is a family of pairwise disjoint relatively open subsets of $Y$. Choosing for each $U$ in $\mathscr{U}$ an open subset $U^{\prime}$ of $X$ for which $U^{\prime} \cap Y=U$, we see that the family $\left\{U^{\prime}: U \in \mathscr{U}\right\}$ is a family of pairwise disjoint open subsets of $X$. Thus $o Y \leqq o X$. The reverse inequality is trivial, since disjoint, nonvoid, open subsets of $X$ have intersections with $Y$ which are disjoint, nonvoid, and relatively open.

4. The nonbounds for $|C(X)|$. In this section we construct a number of spaces for which $|C(X)|$ exceeds $(\delta X)^{o X},(\pi w X)^{w c X}$, and $(w X)^{p X}$, respectively. Those will establish all the " $F$ " entries in Table 1.3. In constructing these spaces, we have avoided (at some loss of brevity) using special arithmetic properties of particular small cardinal numbers; our aim is to show that the smallest cardinal number appearing in any computation may be prescribed in advance, and hence may be as large as desired. We take further pains to point out how the cardinal numbers involved can be minimized, while the desired inequality is still achieved.

4.1 EXample. A space $X$ for which $|C(X)|>(\delta X)^{\circ X}$.

Given an infinite cardinal number $\mathfrak{m}$, let $\left\{X_{\alpha}\right\}_{\alpha \in A}$ be a collection of spaces for each of which $\delta X_{\alpha}=\mathfrak{m}$ and set $X=\Gamma I_{\alpha \in A} X_{\alpha}$. If $A$ is chosen so that $|A|=2^{2^{\mathfrak{m}}}$ then according to 3.1 we have $\delta X \leqq 2^{\mathfrak{m}}$, while from 3.2 we have $o X \leqq \mathfrak{m}$ (and equality in case for at least one $\alpha$ the space $X_{\alpha}$ was chosen so that $\left.o X_{\alpha}=\mathfrak{m}\right)$. To see that $|C(X)| \geqq 2^{2^{\mathfrak{m}}}$, select for each $\alpha$ in $A$ a pair $\left(p_{\alpha}, q_{\alpha}\right)$ of points in $X_{\alpha}$ and an $f_{\alpha}$ in $C\left(X_{\alpha}\right)$ for which $f_{\alpha}\left(p_{\alpha}\right) \neq f_{\alpha}\left(q_{\alpha}\right)$. Then $\left\{f_{\alpha} \circ \pi_{\alpha}: \alpha \in A\right\}$ is a family of $2^{2^{\mathfrak{m}}}$ distinct continuous functions on $X$. Since $|C(X)| \leqq 2^{\delta X}$ we have, in fact,

$$
|C(X)|=2^{2^{\mathfrak{m}}}>2^{\mathfrak{m}}=\left(2^{\mathfrak{m}}\right)^{\mathfrak{m}}=(\delta X)^{o X} .
$$

The construction just given yields spaces for which $|C(X)|>(\delta X)^{o X}$, and for which $\delta X$ and $o X$ are as large as desired, $2^{\mathfrak{m}}$ and $\mathfrak{m}$, respectively. Note that by taking $\mathfrak{m}=\boldsymbol{\aleph}_{0}$, we achieve an example with smallest possible $o X$, and if the continuum hypothesis is assumed, with smallest possible $\delta X$ (for no separable space can have $\left.|C(X)|>\boldsymbol{N}_{0}^{\aleph_{0}}=2 \boldsymbol{N}_{0}\right)$.

4.2 EXAmple. A space $X$ for which $|C(X)|>(\pi w X)^{w c X}$.

Given an infinite cardinal $\mathfrak{m}$, let $\mathfrak{t}=2^{2^{\mathfrak{m}}}$ and let $D$ and $E$ be disjoint discrete spaces with, respectively, $\mathfrak{m}$ and $\mathfrak{t}$ points. Observe that in $\beta E$, the Stone-Čech compactification of $E,\{\{e\}: e \in E\}$ is a minimal $\pi$-base. Let $X$ be the disjoint union of $D$ and $\beta E$. Evidently

$$
\pi w X=\pi w D+\pi w \beta E=\mathfrak{m}+\mathfrak{t}=\mathfrak{l}
$$

and

$$
w c X=w c D+w c \beta E=\mathfrak{m}+\boldsymbol{\aleph}_{0}=\mathfrak{m} .
$$

Now, from the characteristic property of $\beta E$ [GJ, 6.4], the characteristic function of each subset of $E$ extends over $\beta E$, so that $\beta E$, and hence $X$, supports at least (and at most) $2^{t}$ continuous functions. Thus,

$$
|C(X)|=2^{\mathfrak{t}}>\mathfrak{t}=\mathfrak{l}^{\mathfrak{m}}=(\pi w X)^{w c X} .
$$


While the above construction yields a space with $|C(X)|>(\pi w X)^{w c X}$ for which $w c X=\mathfrak{m}=\boldsymbol{\aleph}_{0}$, the example is not compact-compactness is a strong form of " $w c X=\boldsymbol{\aleph}_{0}$ "- and the cardinal number $\pi w X$ is large relative to $w c X$. But even if these are defects, they can be remedied easily.

4.3 EXAMPLe. A compact space $X$ for which $\pi w X=2^{\aleph_{0}}$ and $|C(X)|>(\pi w X)^{w c X}$. Let $D$ be discrete with $|D|=2 \aleph_{0}$, and let $X=\beta D$. As in 4.2,

$$
|C(X)|=2^{2 \aleph_{0}}>2^{\aleph_{0}}=\left(2^{\aleph_{0}}\right)^{\aleph_{0}}=(\pi w X)^{w c X} .
$$

Note that a space of countable $\pi$-weight is separable, so that 4.3 achieves the minimal $\pi$-weight, if the continuum hypothesis is assumed.

4.4 EXAmple. A space $X$ for which $|C(X)|>(w X)^{p X}$.

Given the infinite cardinal $\mathfrak{m}$, let $\mathfrak{n}$ be the cardinal successor of $\mathfrak{m}$, and $\mathfrak{t}=2^{2^{\mathfrak{n}}}$. Let $D$ be the discrete space of $t$ points, and let $X$ be $D$ together with each point of $\beta D$ which is in the closure (in $\beta D$ ) of some subset of $D$ of cardinal $\mathfrak{n}$, but in the closure of no subset of $D$ of cardinal $\leqq \mathfrak{m}$. (Notice that if $E \subset D$ and $|E|=\mathfrak{n}$ then

$$
\left\{\mathrm{cl}_{\beta D} S: S \subset E \text { and }|E-S| \leqq \mathfrak{m}\right\}
$$

is a collection of closed subsets of $\mathrm{cl}_{\beta D} E$ with the finite intersection property. Thus $X \backslash D \neq \varnothing$; indeed, $\left(\mathrm{cl}_{\beta D} E\right) \backslash D \neq \varnothing$ whenever $E \subset D$ and $|E|=\mathfrak{n}$.)

We want to prove that $w X \leqq \mathfrak{f}$ and $p X=\mathfrak{m}$.

For the first, set

$$
Y=D \cup \bigcup\left\{\operatorname{cl}_{\beta D} E: E \subset D \text { and }|E|=\mathfrak{n}\right\} .
$$

For $E \subset D, \mathrm{cl}_{\beta D} E$ is open in $\beta D$, so that $w Y \leqq \sum\left\{w \mathrm{cl}_{\beta D} E: E \subset D\right.$ and $\left.|E|=\mathfrak{n}\right\}$. And $w \mathrm{cl}_{\beta D} E \leqq 2^{|E|}$ whenever $E \subset D$ (because $w \leqq 2^{\delta}$ ), so we have

$$
w X \leqq w Y \leqq 2^{\mathfrak{n}} \cdot \mathfrak{t}^{\mathfrak{n}}=\mathfrak{t}^{\mathfrak{n}}=\mathfrak{t} .
$$

Now for each subset $E$ of $D$ with $|E|=\mathfrak{m}$ the family $\{\{x\}: x \in E\}$ is a locally finite family of open sets in $X$, so that $p X \geqq \mathfrak{m}$. If $p X>\mathfrak{m}$, then there is a locally finite family $\mathscr{U}$ of open sets in $X$, with $|\mathscr{U}|=\mathfrak{n}$. Let $E$ be a set obtained by picking one point of $D$ from each $U \in \mathscr{U}$. Evidently, $|E|=\mathfrak{n}$. We established above that $\left(\left(\mathrm{cl}_{B D} E\right)-D\right) \cap X \neq \varnothing$. But $E$ is closed in $X$ because $\mathscr{U}$ is locally finite, and we have a contradiction.

Finally, $\mid C(D)) \mid=2^{\mathfrak{t}}$, and $|C(D)|=|C(X)|$ because $X$ is between $D$ and $\beta D$. Thus,

$$
|C(X)|=2^{\mathfrak{l}}>\mathfrak{t}=\mathfrak{l}^{\mathfrak{m}} \geqq(w X)^{p X} .
$$

Again, we shall construct an example to the effect of 4.4 but with $p X=\boldsymbol{\aleph}_{0}$ "strongly," and with $w X$ minimal provided the continuum hypothesis is assumed.

The spaces for which each locally finite family of open sets is finite are the pseudocompact ones, i.e., the spaces on which each real-valued continuous function is bounded. See [K] or [BCM, Theorem 3] for a proof of this. 
4.5 EXAmple. A pseudocompact space $X$ for which $w X=2^{\kappa_{0}}$ and $|C(X)|$ $>(w X)^{p X}$.

Let $D$ be discrete of cardinal $2 \aleph_{0}$, and let

$$
X=D \cup \cup\left\{\operatorname{cl}_{\beta D} E: E \subset D \text { and }|E|=\aleph_{0}\right\} .
$$

As in 4.4, $w X \leqq \sum\left\{w \mathrm{cl}_{\beta D} E: E \subset D\right.$ and $\left.|E|=\boldsymbol{\aleph}_{0}\right\}$, and for each $E, w \mathrm{cl}_{\beta D} E \leqq 2 \aleph^{\circ}$. There are $|D|^{\aleph_{0}}$ such $E$ 's, and $w X=2{ }^{\aleph_{0}}$ follows. If $f$ is an unbounded function in $C(X)$, then for each $n$ there is $x_{n} \in D$ with $\left|f\left(x_{n}\right)\right| \geqq n$. The set $E=\left\{x_{n}: n=1,2, \ldots\right\}$ has an accumulation point in $X$, at which $f$ cannot be continuous. So $X$ is pseudocompact. Finally, $|C(X)|=2^{2 \aleph_{0}}$ (as in 4.4), which exceeds $\left(2 \aleph_{0}\right)^{\aleph_{0}}=(w X)^{p X}$.

Observe that by replacing in $4.52 \aleph_{0}$ by any $\mathfrak{m}$ for which $\mathfrak{m}^{\aleph_{0}}<2^{\mathfrak{m}}$, a pseudocompact example $X$ is obtained, with $w X=\mathfrak{m}^{\aleph_{0}}$.

5. The sharpness of the inequality $|C(X)| \leqq(w X)^{w c X}$. The results and examples of the preceding sections show that $(w X)^{w c X}$ is the optimal upper bound for $|C(X)|$ among the numbers we are considering here. However, one can question the sharpness of the estimate in several other senses.

For example, one might ask: (a) Is $w X$ always the least infinite cardinal $\mathfrak{m}$ such that $|C(\dot{X})| \leqq \mathfrak{m}^{w c X}$ ? (b) Is $w c X$ always the least infinite cardinal $\mathfrak{n}$ such that $|C(X)| \leqq(w X)^{\mathfrak{n}}$ ? Spaces for which the answers are in the negative are easy to find. For (a), let $X$ be the discrete space with $2 \aleph_{0}$ points. Here $w X=w c X=2{ }^{\aleph_{0}}$, while

$$
(w X)^{w c X}=\left(2 \aleph_{0}\right)^{2 \aleph_{0}}=\left(\boldsymbol{\aleph}_{0}\right)^{2 \aleph_{0}}=\left(\boldsymbol{\aleph}_{0}\right)^{w c X} .
$$

For (b), let $X$ be the product of $2^{\boldsymbol{k}_{1}}$ discrete spaces each with $\boldsymbol{\aleph}_{1}$ points. Evidently, $w X=2^{\aleph_{1}}$; using 3.2, $w c X=\aleph_{1}$. Thus

$$
(w X)^{w c X}=\left(2^{\aleph_{1}}\right)^{\aleph_{1}}=\left(2^{\aleph_{1}}\right)^{\aleph_{0}}=(w X)^{\aleph_{0}} .
$$

What this shows, of course, is that the questions (a) and (b) are not really questions about topological spaces, but rather questions about cardinal arithmetic.

We turn our attention to the question of whether the inequality " $|C(X)|$ $\leqq(w X)^{w c X}$ " can ever be strict. The comment of the preceding paragraph seems to apply here as well, or at least to our approach. We shall construct a very simple space for which the inequality is strict, based on the following hypothesis.

5.1. There are pairs $(\mathfrak{m}, \mathfrak{t})$ of cardinal numbers which satisfy $\mathfrak{m}^{\mathfrak{t}}>\mathfrak{m} \boldsymbol{N}_{0} \geqq \mathfrak{m} \geqq 2 \mathfrak{t}$.

We shall show below that 5.1 is implied by a nontrivial hypothesis which in strength lies between the continuum hypothesis and the generalized continuum hypothesis. For the nonce, we take a pair $(\mathfrak{m}, \mathfrak{t})$ satisfying 5.1 , and construct our example.

5.2 Example. A space $X$ for which $|C(X)|<(w X)^{w c X}$.

Given $(\mathfrak{m}, \mathfrak{t})$ as in 5.1 , let $X$ be the disjoint union of a compact space $X_{1}$ of weight $\mathfrak{m}$ (e.g., the one-point compactification of the discrete space with $\mathfrak{m}$ points) and a discrete space $X_{2}$ with $\mathfrak{t}$ points. Evidently $w X=w X_{1}+w X_{2}=\mathfrak{m}+\mathfrak{t}=\mathfrak{m}$, and 
$w c X=w c X_{1}+w c X_{2}=\aleph_{0}+\mathfrak{l}=\mathfrak{t}$. And, $|C(X)|=\left|C\left(X_{1}\right) \times C\left(X_{2}\right)\right|=\left|C\left(X_{1}\right)\right| \cdot\left|C\left(X_{2}\right)\right|$. From $2.2,\left|C\left(X_{1}\right)\right| \leqq \mathfrak{m}^{\aleph_{0}}$; and, of course, $\left|C\left(X_{2}\right)\right|=2^{\mathfrak{t}}$. Thus

$$
|C(X)| \leqq \mathfrak{m}^{\aleph_{0}} \cdot 2^{\mathfrak{f}}=\mathfrak{m}^{\aleph_{0}}<\mathfrak{m}^{\mathfrak{l}}=(w X)^{w c X} .
$$

The remainder of this section is devoted to determining reasonably weak hypotheses sufficient to guarantee the validity of hypothesis 5.1.

5.3 Discussion. It is easy, using the König-Zermelo theorem and arguing as in [B, p. $125(5)$ ], to find for each $\mathfrak{t}$ a cardinal $\mathfrak{m}$ as large as desired for which $\mathfrak{m}^{\mathfrak{t}}>\mathfrak{m}$. Specifically, if $\left\{\mathfrak{m}_{\alpha}: \alpha<\beta\right\}$ is any family of cardinal numbers order-isomorphic with the set of ordinals preceding the smallest ordinal number $\beta$ of cardinality $\mathfrak{f}$, there exists $\mathfrak{m}$ for which $\mathfrak{m}^{\mathfrak{t}}>\mathfrak{m} \geqq 2^{\mathfrak{f}}$, and for which $\mathfrak{m}$ is the supremum of countably many cardinal numbers each less than $\mathfrak{m}$ if and only if $\mathfrak{f}$ is the supremum of countably many cardinals each less than $f$. It turns out that with an additional hypothesis on $\mathfrak{m}$, which hypothesis we shall call $*(\mathfrak{m})$, the denial of this lastcited condition on $\mathfrak{m}$ (equivalently on $\mathfrak{f}$ ) suffices to guarantee that $\mathfrak{m}=\mathfrak{m}^{\aleph_{0}}$, thus guaranteeing 5.1. We now state hypothesis $*(\mathfrak{m})$ and offer the indicated argument in 5.3, though this argument has been used in both [B] and [T] to yield similar exponential information.

HYPOTHESIS $*(\mathfrak{m})$. There is no cardinal number $\mathfrak{p}$ for which $\mathfrak{p}<\mathfrak{m}<\mathfrak{p}^{N_{0}}$.

5.4 THEOREM. If $\mathfrak{m}^{\aleph_{0}}>\mathfrak{m} \geqq \aleph_{0}$, and if $*(\mathfrak{m})$ holds, then $\mathfrak{m}$ is the sum of countably many smaller cardinals.

Proof. Let $\omega_{\mathfrak{m}}$ be the first ordinal of cardinality $\mathfrak{m}$. If $\mathfrak{m}$ is not the sum of countably many smaller cardinals, then $\left\{\alpha: \alpha<\omega_{\mathfrak{m}}\right\}$ has no countable cofinal subset. Thus, writing

$$
\mathscr{C}(\alpha) \equiv\left\{S: S \subset\{\beta: \beta<\alpha\} \text { and }|S|=\boldsymbol{\aleph}_{0}\right\}
$$

for each ordinal $\alpha \leqq \omega_{\mathfrak{m}}$, we have

$$
\mathscr{C}\left(\omega_{\mathfrak{m}}\right) \subset \bigcup\left\{\mathscr{C}(\alpha): \alpha<\omega_{\mathfrak{m}}\right\} .
$$

Hence,

$$
\begin{aligned}
\mathfrak{m}^{\aleph_{0}} & =\left|\mathscr{C}\left(\omega_{\mathfrak{m}}\right)\right| \leqq \sum\left\{|\mathscr{C}(\alpha)|: \alpha<\omega_{\mathfrak{m}}\right\} \\
& \leqq \sum\left\{|\alpha|^{\aleph_{0}}: \alpha<\omega_{\mathfrak{m}}\right\} .
\end{aligned}
$$

Now $*(\mathfrak{m})$ implies that $|\alpha|^{\aleph_{0}} \leqq \mathfrak{m}$ for each $\alpha<\omega_{\mathfrak{m}}$, and $\mathfrak{m}^{\aleph_{0}} \leqq \mathfrak{m} \cdot \mathfrak{m}=\mathfrak{m}$ results.

The following result simply summarizes what has been written. No proof is required, since (a) is given by 5.3 and 5.4 and (b) by (a) and 5.2.

5.5 TheOREM. Suppose that $*(\mathfrak{m})$ holds for each cardinal number $\mathfrak{m}$. Let $\mathfrak{t}$ be any cardinal number not the sum of countably many smaller cardinals-e.g., let $\mathfrak{f}$ have an immediate cardinal predecessor-and determine $\mathfrak{m}$ from $\mathfrak{t}$ as in 5.3. Then

(a) $\mathfrak{m}^{\mathfrak{t}}>\mathfrak{m}^{\aleph_{0}}=\mathfrak{m} \geqq 2^{\mathfrak{t}}$, and

(b) there is a space $X$ for which $|C(X)|=\mathfrak{m}$ and $w X=\mathfrak{m}$ and $w c X=\mathfrak{t}$. 
5.6 REMARK. A word is in order concerning the strength of the hypothesis $*(\mathfrak{m})$. It is shown in [B, p. 157] that the generalized continuum hypothesis is equivalent to the condition "for each cardinal number $m$, the inequality $m<\mathfrak{m}^{\mathfrak{t}}$ holds only if $\mathfrak{m}$ is the sum of $\mathfrak{t}$ or fewer cardinal each less than $\mathfrak{m}$." In particular, our condition that $*(\mathfrak{m})$ holds for each cardinal number $\mathfrak{m}$ follows from the generalized continuum hypothesis. The special case $*\left(\boldsymbol{\aleph}_{1}\right)$, of course, is equivalent to the continuum hypothesis $\aleph_{1}=2^{\aleph_{0}}$.

6. Metric spaces. The generally complex situation surrounding the numbers $p X-w X$ of 1.1 , and their relation to $|C(X)|$, simplifies for metric spaces as much as conceivable.

6.1 Proposition. If $X$ is metrizable, then the six numbers of 1.1 are equal, and $|C(X)|=2^{\delta X}$.

Proof. Consider briefly the "covering number," $c X$, the least $m$ for which each open cover has a subcover with $\mathfrak{m}$ or fewer members. For metrizable $X, c X=w X$, as is shown in [En, p. 176]. By 1.2, the six numbers of 1.1 will be equal provided that $p X=c X$. Indeed, this equality holds for each paracompact space $X$, hence surely whenever $X$ is metric. To see this let $\mathscr{U}$ be an open cover for the paracompact space $X$, and choose a locally finite open refinement $\mathscr{V}$ of $\mathscr{U}$; clearly $|\mathscr{V}| \leqq p X$. A subcover of $\mathscr{U}$ of cardinal $|\mathscr{V}|$ or less is constructed by picking, for each $V \in \mathscr{V}$, a $U_{V} \in \mathscr{U}$ with $V \subset U_{V}$.

Since for metrizable $X, \delta X=w X$ (e.g., [En, p. 176]), and $|C(X)| \leqq 2^{\delta X}$ always holds, it remains simply to show that $|C(X)| \geqq 2^{\delta X}$. It is known that the metrizable space $X$ admits a collection $\mathscr{O l}$ of nonvoid, pairwise disjoint, open subsets for which $|\mathscr{U}|=\delta X$. (The statement appears in [deG]. If $\mathscr{B}=\bigcup_{n \in N} \mathscr{B}_{n}$ is a basis for $X$, with each family $\mathscr{B}_{n}$ a collection of pairwise disjoint open sets, then either $o X=\left|\mathscr{B}_{n}\right|$ for some $n$ or the number $o X$ is the supremum of countably many smaller cardinals. In this latter case, even if $o X$ has a weakly inaccessible predecessor, the argument given in [Ef, Lemma 1] suffices to complete the proof.) Now for each subset $\mathscr{V}$ of $\mathscr{U}$ we set $f_{\mathscr{V}}(x)=\rho(x, \cup \mathscr{V})$ (with, say, $f_{\phi} \equiv 1$ on $X$ ). Distinct subsets of $\mathscr{U}$ yield distinct continuous functions, for if $p \in \bigcup \mathscr{V}_{1} \backslash \cup \mathscr{V}_{2}$ then

$$
f_{\mathscr{V}_{1}}(p)=0<f_{\mathscr{V}_{2}}(p)
$$

since $p \notin \mathrm{cl}_{X} \cup \mathscr{V}_{2} ;$ hence $|C(X)| \geqq 2^{\delta X}$.

7. Remarks on the weight of a Stone-Čech compactification. First of all, $|C(X)|$ can be calculated exactly in terms of $w \beta X$, using results of Smirnov and Kruse. The result is that $|C(X)|=(w \beta X)^{\aleph_{0}}$, and the argument is as follows.

$C^{*}(X)$, the subset of $C(X)$ consisting of bounded functions, can be normed via $\|f\|=\sup \{f(x): x \in X\}$. Smirnov has shown in [S] that, giving $C^{*}(X)$ the associated topology, $\delta C^{*}(X)=w \beta X$. Since the sequences from a dense subspace of $C^{*}(X)$ determine uniquely all functions in $C^{*}(X)$, it follows that $\left|C^{*}(X)\right| \leqq\left(\delta C^{*}(X)\right)^{\aleph_{0}}$ 
$=(w \beta X)^{\aleph_{0}}$. Now, Kruse has shown in [Ku] that if $E$ is any complete normed linear space, then $|E|=|E|^{*}$. Applying this to $C^{*}(X)$, and observing that $|C(X)|$ $=\left|C^{*}(X)\right|$, we have $|C(X)|=(w \beta X)^{\aleph_{0}}$.

In terms of $X, w \beta X$ can be estimated in at least two ways. First, $\delta X \leqq w X \leqq w \beta X$ $\leqq 2^{\delta \beta X} \leqq 2^{\delta X}$, which assuming the generalized continuum hypothesis equates $w \beta X$ with either $\delta X$ or $2^{\delta X}$. Alternatively, we can argue as follows. $C^{*}(X)$ and $C(\beta X)$ are isomorphic, hence equipotent, so that

$$
w \beta X \leqq|C(\beta X)|=\left|C^{*}(X)\right|=|C(X)| \leqq(w X)^{w c X} \leqq(w \beta X)^{w c X} .
$$

(The first inequality was remarked on in $\S 2$.) Exponentiating by $w c X$, we obtain the following simple result.

\subsection{Proposition. For any $X,(w X)^{w c X}=|C(X)|^{w c X}=(w \beta X)^{w c X}$.}

These equalities for Lindelöf spaces were noted in $\left[\mathrm{H}_{1}\right]$.

8. The covering number. Once again, $c X$ is the least $\mathrm{m}$ for which each open cover of $X$ has a subcover with $m$ or fewer members. We examine here its use in estimating $|C(X)|$. The only easily noticed general inequalities relating $c X$ with the numbers of 1.1 are: $w c X \leqq c X \leqq w X$. (In particular, the usual examples of a separable non-Lindelöf space, and of a Lindelöf nonseparable space, show that $c X$ and $\delta X$ are generally incomparable.) For paracompact spaces $X$ always $p X=c X$, as was pointed out in the proof of 6.1. From Example 4.2, then, $|C(X)|>(\pi w X)^{c X}$ can occur, which also disposes of $(\delta X)^{c X},(o X)^{c X}$, and $(p X)^{c X}$ as candidates for upper bounds. Of course, $|C(X)| \leqq(w X)^{c X}$ is valid, using 2.2. Since $|C(X)| \leqq 2^{\delta X}$, the numbers $(c X)^{\delta X},(c X)^{\pi w X},(c X)^{w X}$ also are upper bounds for $|C(X)|$. Finally, $|C(X)|>(c X)^{o X}\left(\geqq(c X)^{w c X} \geqq(c X)^{p X}\right)$ can occur: use Example 4.1 with each $X_{\alpha}$ compact, so that $c X=\boldsymbol{\aleph}_{0}$. This exhausts the numbers involving $c X$ that we care to consider.

\section{REFERENCES}

[B] Heinz Bachmann, Transfinite Zahlen, Ergebnisse der Mathematik und ihrer Grenzgebiete, Heft 1, Springer-Verlag, Berlin and New York, 1955. MR 17, 134.

[BCM] R. W. Bagley, E. H. Connell and J. D. McKnight, Jr., On properties characterizing pseudo-compact spaces, Proc. Amer. Math. Soc. 9 (1958), 500-506. MR 20 \#3523.

[CHN] W. W. Comfort, Neil Hindman and S. Negrepontis, $F^{\prime}$-spaces and their product with P-spaces, Pacific J. Math. 28 (1969), 489-502.

[deG] J. deGroot, Discrete subspaces of Hausdorff spaces, Bull. Acad. Polon. Sci. Sér. Sci. Math. Astronom. Phys. 13 (1965), 537-544. MR 35 \#956.

[Ef] B. Efimov, Extremally disconnected bicompacta whose $\pi$-weight is the continuum, Dokl. Akad. Nauk SSSR 183 (1968), 511-514=Soviet Math. Dokl. 9 (1968), 1404-1407. MR 38 \#5172.

[En] R. Engelking, Outline of general topology, North-Holland, Amsterdam and Interscience, New York, 1968. MR 37 \#5836.

[ET] P. Erdös and A. Tarski, On families of mutually exclusive sets, Ann. of Math. (2) 44 (1943), 315-329. MR 4, 269. 
[F] Zdeněk Frolík, Generalizations of compact and Lindelöf spaces, Czechoslovak Math. J. 9 (84) (1959), 172-217. (Russian) MR 21 \#3821.

[GJ] Leonard Gillman and Meyer Jerison, Rings of continuous functions, The University Series in Higher Math., Van Nostrand, Princeton, N. J., 1960. MR 22 \#6994.

$\left[\mathrm{H}_{1}\right]$ Anthony W. Hager, Approximation of real continuous functions on Lindelöf spaces, Proc. Amer. Math. Soc. 22 (1969), 156-163.

$\left[\mathrm{H}_{2}\right]-$ - Projections of zero-sets (and the fine uniformity on a product), Trans. Amer. Math. Soc. 140 (1969), 87-94.

[He] Edwin Hewitt, A remark on density characters, Bull. Amer. Math. Soc. 52 (1946), 641-643. MR 8, 139.

[Hi] Neil Hindman, On P-like spaces and their product with P-spaces, Doctoral Dissertation, Wesleyan University, Middletown, Conn., 1969.

[I] J. R. Isbell, Uniform spaces, Math. Surveys, no. 12, Amer. Math. Soc., Providence, R. I., 1964. MR 30 \#561.

[K] J. Kerstan, Zur charakterisierung der pseudokompakten Räume, Math. Nachr. 16 (1957), 289-293. MR 19, 1069.

[Ku] Arthur H. Kruse, Badly incomplete normed linear spaces, Math. Z. 83 (1964), 314-320. MR 29 \#3859.

[M] Edward Marczewski, Séparabilité et multiplication cartésienne des espaces topologiques, Fund. Math. 34 (1947), 127-143. MR 9, 98.

[N] Norman Noble, Products with closed projections, Trans. Amer. Math. Soc. 140 (1969), 381-391.

[O] J. C. Oxtoby, Cartesian products of Baire spaces, Fund. Math. 49 (1960/61), 157-166. MR 25 \#4055; MR 26, 1543.

[Pz] E. S. Pondiczery, Power problems in abstract spaces, Duke Math. J. 11 (1944), 835-837. MR 6, 119.

[Pm] V. I. Ponomarev, Spaces co-absolute with metric spaces, Uspehi Mat. Nauk 21 (1966), no. 4 (130), 101-132=Russian Math. Surveys 21 (1966), no. 4, 87-113. MR 34 \#788.

[RS] K. A. Ross and A. H. Stone, Products of separable spaces, Amer. Math. Monthly 71 (1964), 398-403. MR 29 \#1611.

$\left[\breve{S}_{1}\right]$ N. A. Sanin, On intersection of open subsets in the product of topological spaces, Dokl. Akad. Nauk SSSR 53 (1946), 499-501. (Russian) MR 8, 334.

$\left[\check{\mathrm{S}}_{2}\right]-\ldots$, On the product of topological spaces, Trudy Mat. Inst. Steklov. 24 (1948). (Russian) MR 10, 287.

[S] Yu. M. Smirnov, On the weight of the ring of bounded continuous functions over a normal space, Mat. Sb. 30 (72) (1952), 213-218. (Russian) MR 14, 70.

[T] Alfred Tarski, Quelques théorèmes sur les alelphs, Fund. Math. 7 (1925), 1-14.

WESLEYAN UNIVERSITY, Middletown, Connecticut 06457 